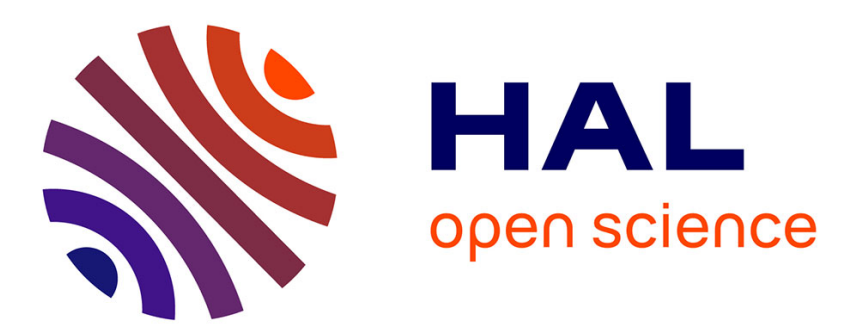

\title{
A model for stratified gas-liquid turbulent flow in ducts of arbitrary cross-section
}

\author{
J.M. Fitremann, J.M. Rosant
}

\section{To cite this version:}

J.M. Fitremann, J.M. Rosant. A model for stratified gas-liquid turbulent flow in ducts of arbitrary cross-section. Revue de Physique Appliquée, 1981, 16 (3), pp.93-103. 10.1051/rphysap:0198100160309300 . jpa-00244905

\section{HAL Id: jpa-00244905 https://hal.science/jpa-00244905}

Submitted on 1 Jan 1981

HAL is a multi-disciplinary open access archive for the deposit and dissemination of scientific research documents, whether they are published or not. The documents may come from teaching and research institutions in France or abroad, or from public or private research centers.
L'archive ouverte pluridisciplinaire HAL, est destinée au dépôt et à la diffusion de documents scientifiques de niveau recherche, publiés ou non, émanant des établissements d'enseignement et de recherche français ou étrangers, des laboratoires publics ou privés. 


\title{
A model for stratified gas-liquid turbulent flow in ducts of arbitrary cross-section
}

\author{
J. M. Fitremann and J. M. Rosant \\ GIS ENSM-CNRS, 1, rue de la Noë, 44072 Nantes Cedex, France
}

(Reçu le 19 octobre 1979, révisé le 8 février 1980, accepté le 8 décembre 1980)

Résumé. - Nous proposons une théorie permettant le calcul du gradient de pression et du taux de liquide d'un écoulement diphasique gaz-liquide établi en configuration stratifiée dans une conduite cylindrique de section et d'inclinaison quelconques et son application à une conduite faiblement inclinée sur l'horizontale.

La validité est limitée aux valeurs des nombres de Reynolds pour lesquels l'écoulement est turbulent dans les deux phases. L'entraînement de gouttelettes dans la phase gazeuse ou de bulles dans la phase liquide n'est pas pris en compte. La comparaison du calcul et de valeurs expérimentales obtenues avec l'air et l'eau dans des conduites circulaires et rectangulaires est très satisfaisante.

\begin{abstract}
We propose a theory to calculate the pressure gradient and the liquid fraction in two-phase gasliquid fully developed stratified flow in a cylindrical duct of arbitrary cross-section and slope and its application to near horizontal ducts.

Its validity is limited to Reynolds numbers high enough to ensure turbulent flow in both phases. Droplet entrainment or bubble inclusion have not been taken into account. The comparison between calculations and experimental values obtained with air and water in circular and rectangular ducts is very satisfactory.
\end{abstract}

Nomenclature. - TWO-PHASE VARIABLES.

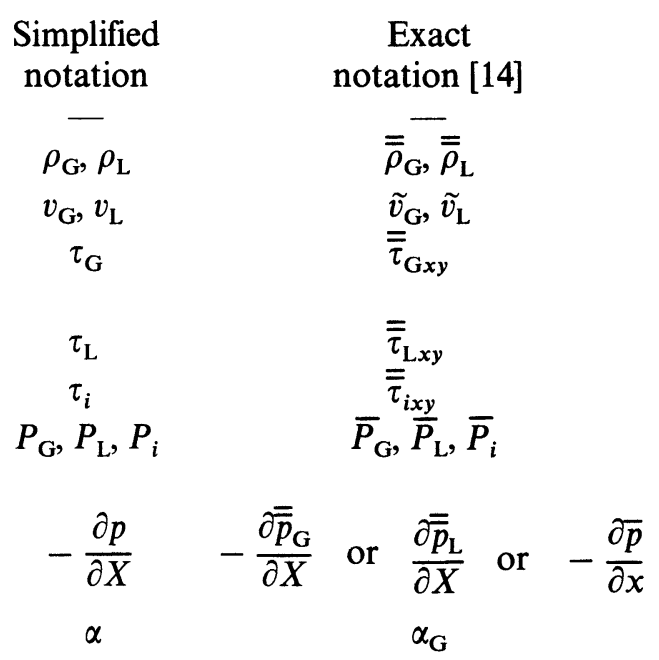

Definition

densities statistical average (gas, liquid) mass average phase velocities (gas, liquid) statistical average of streamwise component of tangential stress at gas-wall boundary

id. at liquid-wall boundary

$i d$. at gas-liquid boundary

statistical average of gas-wall, liquid-wall and gas-liquid perimeters respectively

statistical average of streamwise component of pressure gradient statistical average of the gas characteristic function
OTHER QUANTITIES.

$A$

$B_{\mathrm{L}}, B_{1}, B_{2}$

$B_{2}^{\prime}$
$C_{0}, C_{1}, C_{2}$

$C_{L}, C_{G_{1}}, C_{G_{2}}$ universal consta
velocity profile second constants for logarithmic velo- $D_{0}, D_{1}, D_{2}$ city profiles, roughness dependent, $D_{\mathrm{H}}=4 S / P$ Nikuradse's constant, universal functions of $\alpha$ for the friction laws,

logarithmic velocity defect laws constants,

integrals of the velocity defect laws, hydraulic diameter of the cross-section, 


\begin{tabular}{|c|c|}
\hline$f_{\mathrm{L}}, f_{\mathrm{G}}, f_{i}$ & $\begin{array}{l}\text { friction coefficients, defined in (15), } \\
\text { (23) and (28) }\end{array}$ \\
\hline & gravity constant, \\
\hline$G_{\mathrm{L}}, G_{1}, G_{2}$ & velocity defect laws, \\
\hline $\begin{array}{l}L, L_{1}, L_{2} \\
P\end{array}$ & $\begin{array}{l}\text { length scales for velocity defect laws, } \\
\text { perimeter of cross-section, }\end{array}$ \\
\hline$Q_{\mathrm{L}}, Q_{\mathrm{G}}$ & $\begin{array}{l}\text { mass flow rates of the liquid, gas } \\
\text { respectively, }\end{array}$ \\
\hline $\begin{array}{l}R e_{\mathrm{L}}, R e_{\mathrm{G}} \\
S\end{array}$ & $\begin{array}{l}\text { liquid and gas Reynolds numbers, } \\
\text { cross-section area, }\end{array}$ \\
\hline$S_{1}, S_{2}$ & $\begin{array}{l}\text { parts of the gas filled cross-section, } \\
\text { wall influenced and interface influenc- } \\
\text { ed respectively, }\end{array}$ \\
\hline$S_{\mathrm{G}}$ & gas cross-section (defined in A.3), \\
\hline $\begin{array}{l}S_{\mathrm{L}}=S-S_{\mathrm{G}} \\
U_{\mathrm{L}}, U_{\mathrm{G}}\end{array}$ & $\begin{array}{l}\text { liquid cross-section, } \\
\text { superficial velocities, }\end{array}$ \\
\hline$u_{*}, u_{* \mathrm{G}}, u_{* i}$ & local friction velocities, \\
\hline & $\begin{array}{l}\text { root mean square friction velocities, } \\
\text { slip velocity at mean interface, }\end{array}$ \\
\hline$X, Y, Z$ & $\begin{array}{l}\text { cartesian frame, } X \text { directed along } \\
\text { the flow direction, }\end{array}$ \\
\hline$x, y, z$ & $\begin{array}{l}\text { local boundary coordinates, } y \text { normal } \\
\text { to the boundary, }\end{array}$ \\
\hline $\begin{array}{l}\alpha, \alpha_{L}=1-\alpha \\
\beta\end{array}$ & $\begin{array}{l}\text { gas fraction, liquid fraction, } \\
\text { angle of duct with horizontal, positive } \\
\text { upwards, }\end{array}$ \\
\hline$\varepsilon$ & $\begin{array}{l}\text { roughness height of the wavy inter- } \\
\text { face, }\end{array}$ \\
\hline$\mu_{\mathrm{L}}, \mu_{\mathrm{G}}$ & dynamic viscosities, \\
\hline $\mathrm{L}, v_{\mathrm{G}}$ & kinematic viscosities. \\
\hline
\end{tabular}

1. Introduction. - Stratified turbulent flow of a gas-liquid mixture occurs in many industrial situations and its accurate prediction is of technical and economical importance $\left({ }^{1}\right)$. There is also a current interest in the momentum transfer mechanism at a free surface under various conditions. Finally, of all the two phase flow configurations encountered in practice (Govier and Aziz [1], Bonnin et Fitremann [2]), it has the advantage of being nearest to single phase flow and its mathematical description may throw some light on more general transfer concepts in theoretical two-phase dynamics.

Yu and Sparrow [3] have been able to solve numerically the laminar case and a number of authors have attempted the description of the fully turbulent gasliquid stratified flow (Flanigan [4], Eaton [5], Taitel and Dukler [6], Russel et al. [7], Cheremisinoff and Davis [8], Crouzier [9]). Although some authors have written a correct two-fluid momentum balance equation for the stratified flow case, it appears that heuristic definitions of the various mean values and fric-

$\left({ }^{1}\right)$ Of the many fields of application, oil and gas transport, chemical engineering, and nuclear reactors safety analysis are the most important. Stratified here has the usual meaning in two-phase flow literature, i.e. both phase flow separately. It is opposed to dispersed flow where one phase is bounded by a multiply connected interface. tion coefficients does not give an accurate prediction of the liquid fraction, especially in upward flow, even if by some adjustments they can be made to predict the pressure gradient satisfyingly. A comparison between several methods and experiments can be found in Crouzier [9].

We have thus attempted to describe rationally the main features of high Reynolds number co-current stratified flow using two-phase and turbulent flow concepts as described in the following sections.

The central idea developed hereafter is to use two parameter families for the velocity profiles (law of the wall and velocity defect law) in three distinct regions of the cross section, namely the liquid filled area, a wall influenced gas area and an interface influenced gas area. A method is proposed to reduce the somewhat arbitrary separation of the gas flow into two parts. Using this scheme, it will be shown that both pressure gradients and liquid content can be accurately predicted, once the physical parameters have been correctly chosen.

2. Basic concepts. - We assume throughout that steady and fully developed stratified gas-liquid flow occurs in a cylindrical duct of arbitrary cross-section and slope. That the momentum exchange between phases through wave-interaction process can reach an equilibrium is found from experiments. Thus, time mean values of physical quantities are steady and independent of the streamwise coordinate. Single phase turbulent flow is prevalent in most of the liquid and gas regions and wavy flow in the interaction region.

The treatment of the single phase regions will closely follow the methods used for the prediction of high Reynolds number turbulent flow in ducts of arbitrary cross-section (Fortier [10]). The interaction region will be idealized as follows :

- The wavy interface is replaced by a fictitious steady boundary, the position of which is well defined by the gas-liquid interaction properties and will be called the mean interface.

- The gas flow above the mean interface is simulated by a wall turbulent shear flow over an axially moving rough boundary.

- The mean interface is assumed to be a free surface for the liquid turbulent flow. Actually, the liquid flow can be resolved into two components : one, the bulk flow mainly influenced by the wall stress; the other, the drift flow mainly influenced by the interfacial stress. However, for the application we have in mind i.e. co-current flow, the experiments [16] show that the contribution of the excess velocity in the drift current region to the total flow rate is small enough to be neglected (Fig. 1). Thus, the results derived here can not be applied to countercurrent flow without further refinements. Owing to the lack of circular symmetry of the flow in both phases, secondary flow and variation of shear stress along 

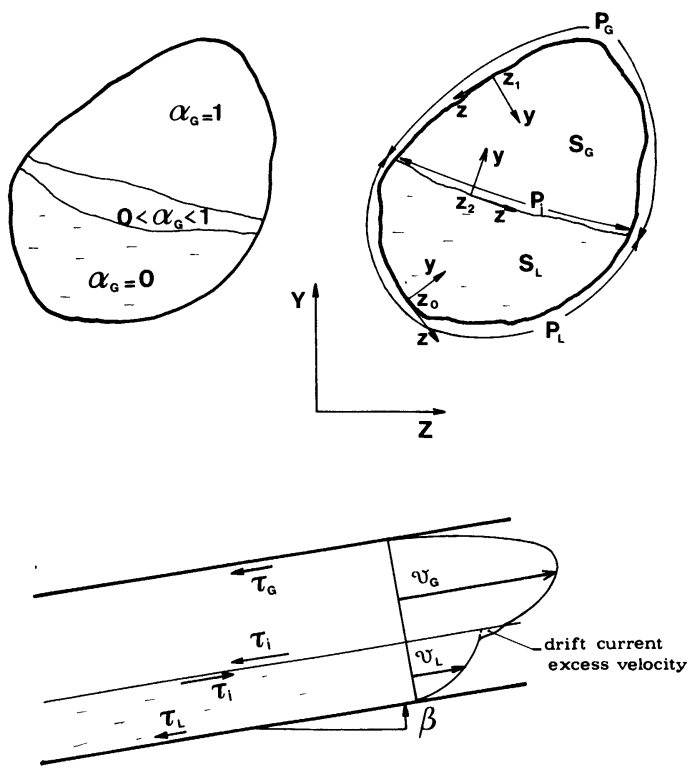

Fig. 1. - Stratified gas-liquid flow. Sketch of cross-section.

the boundaries occur and have to be taken into account.

Wall bounded shear flow properties at high Reynolds number such as described in Hinze [11], Tennekes and Lumley [12] for single phase flow in pipes and channels are however assumed to be relevant, secondary flows being taken into account only through shear stress variation with the transverse coordinates.

Two-phase theorems as derived by Ishii [13], Fitremann [14], Delhaye and Achard [15] are used in their time and cross-section average form. To avoid unnecessary complication of the symbols, only time averaged quantities will be used. The proper definitions of the symbols used are given in detail in the appendix and nomenclature section.

Following the above guidelines, we get some simplification of the phase-interaction process, viz. :

- The exchange of mass through chemical reaction and entrainment of bubbles or droplets is neglected.

- The exchange of momentum between the gas and the liquid is obtained i) through continuity of the normal stress at the mean interface ii) through continuity of the tangential stress at the mean interface. However, strong liquid $\rightarrow$ gas interaction occurs through the wave mechanism modelled here by a rough gas-liquid boundary, and weaker gas $\rightarrow$ liquid interaction owing to the neglect of the contribution of the excess velocity in the drift current zone to the total liquid flow rate.

By a standard procedure in fully developed flow $[11,12]$, it is found that the sum of the hydrostatic pressure and a combination of the normal turbulent stresses is constant in a plane normal to the flow direction and thus the streamwise pressure gradient component is the same for any reference point chosen in the cross section owing to the continuity of the normal stress across the interface $\left({ }^{2}\right)$.

Then, the mean interface is chosen so as to simplify the mass conservation equations, as shown in the appendix, into

$$
\begin{aligned}
& Q_{\mathrm{L}}=\rho_{\mathrm{L}} U_{\mathrm{L}} S=\left\langle v_{\mathrm{L}}\right\rangle_{S_{\mathrm{L}}}(1-\alpha) \rho_{\mathrm{L}} S \\
& Q_{\mathrm{G}}=\rho_{\mathrm{G}} U_{\mathrm{G}} S=\left\langle v_{\mathrm{G}}\right\rangle_{S_{\mathrm{G}}} \alpha \rho_{\mathrm{G}} S
\end{aligned}
$$

where :

$\alpha$ is the cross-section averaged gas time-fraction $S_{\mathrm{G}}=\alpha S$ is the gas filled cross-section area (Fig. 1) $S_{L}=(1-\alpha) S$ is the liquid filled cross-section area and \langle\rangle denotes averaging over the variety indicated by a subscript. (All symbols are properly defined in the appendix and nomenclature section.)

The streamwise momentum balance equations for each phase reads

$$
\begin{aligned}
& -\frac{\partial p}{\partial X}=\frac{P_{\mathrm{L}}}{S_{\mathrm{L}}}\left\langle\tau_{\mathrm{L}}\right\rangle_{P_{\mathrm{L}}}-\frac{P_{i}}{S_{\mathrm{L}}}\left\langle\tau_{i}\right\rangle_{P_{i}}+\rho_{\mathrm{L}} g \sin \beta \\
& -\frac{\partial p}{\partial X}=\frac{P_{\mathrm{G}}}{S_{\mathrm{G}}}\left\langle\tau_{\mathrm{G}}\right\rangle_{P_{\mathrm{G}}}+\frac{P_{i}}{S_{\mathrm{G}}}\left\langle\tau_{i}\right\rangle_{P_{i}}+\rho_{\mathrm{G}} g \sin \beta
\end{aligned}
$$

where $p$ is the mean pressure at any reference point in the cross-section, $P_{\mathrm{L}}, P_{\mathrm{G}}, P_{i}$ are the liquid-wall, gaswall and gas-liquid interface perimeters respectively and $\tau_{\mathrm{L}}, \tau_{\mathrm{G}}, \tau_{i}$ the corresponding shear stress along these boundaries, the signs of which have been chosen so that they are positive in co-current flow with the higher velocity on the gas side when no flow reversal occurs along the duct wall.

By eliminating the pressure gradient between (3) and (4), the balance equation

$$
\begin{aligned}
\frac{P_{\mathrm{L}}}{S_{\mathrm{L}}}\left\langle\tau_{\mathrm{L}}\right\rangle_{P_{\mathrm{L}}}-P_{i}\left(\frac{1}{S_{\mathrm{G}}}\right. & \left.+\frac{1}{S_{\mathrm{L}}}\right)\left\langle\tau_{i}\right\rangle_{P_{i}}-\frac{P_{\mathrm{G}}}{S_{\mathrm{G}}}\left\langle\tau_{\mathrm{G}}\right\rangle_{P_{\mathrm{G}}}+ \\
& +\left(\rho_{\mathrm{L}}-\rho_{\mathrm{G}}\right) g \sin \beta=0
\end{aligned}
$$

is obtained. Phenomenological relations for $\tau_{i}, \tau_{G}, \tau_{L}$ and the mean interface profile are necessary to close the system. $\alpha$ is then readily obtained from equation (5) and the pressure gradient can then be calculated from (3) or (4).

The central idea in our model consists in the distinction of three regions in the cross-section : one is the liquid filled region; the two others are in the gas phase, one where the flow is essentially influenced by the wall, the other where the flow is essentially influenced by the wavy interface (Fig. 1). In each of these regions, the properties of turbulent shear flow at high Reynolds number are assumed to be valid and the solution of the Reynolds equations uniquely determined by the boundary conditions.

${ }^{2}$ ) The pressure itself is not necessarily continuous since there can be a jump of the normal turbulent stress at the mean interface. However in fully developed flow this jump is axially constant and the equality of the pressure gradient in both phases holds. 
3. The liquid phase region. - We assume, as in the treatment of open channel flow, that the turbulent properties of the liquid flow are, apart from a small region near the interface where the drift current occurs, directly influenced by the solid boundary. Local measurements of velocity profiles such as shown in Suzanne [16] indicate that this approximation is valid when the liquid flow rate is high enough to allow the contribution of the excess drift current to the total liquid flow rate to be negligibly small.

We now use, in addition to the main cartesian frame of reference $(X, Y, Z ; X$ streamwise coordinate), curvilinear wall coordinates $(x, y, z)$ so that $y$ is directed along the normal to the wall and $z$ is measured along the wall from some arbitrary origin.

In wall coordinates, the universal velocity profile in the inner layer is written

$$
\frac{v_{\mathrm{L}}}{u_{*}(z)}=F_{\mathrm{L}}\left(\frac{y u_{*}(z)}{v_{\mathrm{L}}}\right)
$$

where

$$
\left[u_{*}(z)\right]^{2}=\frac{\tau_{\mathrm{L}}(z)}{v_{\mathrm{L}}} .
$$

In the core region, dimensional analysis leads to the following expression for the velocity defect law :

$$
\frac{v_{\mathrm{L}}-v_{\mathrm{L} \max }}{u_{* 0}}=G_{\mathrm{L}}\left(\frac{Y}{L}, \frac{Z}{L}\right)
$$

where $u_{* 0}$ is a friction velocity scale and $L$ a length scale of the mean flow. $v_{\mathrm{L} \text { max }}$ is the maximum mean axial velocity of the flow. $G_{\mathrm{L}}$ is a universal function for a given liquid cross-section $S_{\mathrm{L}}$, thus a universal function of $\alpha$ (and $Y, Z$ ) for a given pipe cross-section.

For sufficiently high Reynolds number, both laws are valid in an overlap layer; (6) and (8) then assume the necessary form (in wall coordinates) :

$$
\text { law of the wall } \frac{v_{\mathrm{L}}}{u_{*}(z)}=A \log \frac{y u_{*}(z)}{v_{\mathrm{L}}}+B_{0}
$$

velocity defect law in wall coordinates

$$
\frac{v_{\mathrm{L}}-v_{\mathrm{L} \max }}{u_{*}(z)}=A \log \frac{y}{L}+C_{\mathrm{L}}\left(\frac{z}{L}\right)
$$

(10) actually defines the universal function $C_{\mathrm{L}}$ of $\alpha$ and $z / L$ from $G_{\mathrm{L}} \cdot 1 / A$ is the Von Karman constant. $B_{0}$ depends on the wall roughness as in single phase pipe flow.

Combining (9) and (10) gives

$$
\frac{v_{\mathrm{L} \max }}{u_{*}(z)}=A \log \frac{u_{*}(z) L}{v_{\mathrm{L}}}+B_{0}-C_{L}\left(\frac{z}{L}\right) .
$$

This relation links the variations of $u_{*}(z)$ to the universal function $G_{\mathrm{L}}$ through its image in the wall coordinates $C_{L}$ for a given liquid fraction and crosssection.
If we now chose $u_{* 0}$ such as

$$
\left\langle\tau_{\mathrm{L}}\right\rangle_{P_{\mathrm{L}}}=\rho_{\mathrm{L}} u_{* 0}^{2}=\rho_{\mathrm{L}}\left\langle u_{*}^{2}\right\rangle_{P_{\mathrm{L}}}
$$

using the mean value theorem we are assured of the existence of an abcissae $z_{0}$ on $P_{\mathrm{L}}$ where $u_{*}\left(z_{0}\right)=u_{* 0}$.

(10) can be written at this particular point $z_{0}$ and substracted from (8) to give

$$
\begin{aligned}
\frac{v_{\mathrm{L}}}{u_{* 0}}=\frac{v_{\mathrm{L} \max }}{u_{* 0}}+ & G_{\mathrm{L}}=A \log \frac{u_{* 0} L}{v_{\mathrm{L}}}+ \\
+ & B_{0}-C_{\mathrm{L}}\left(\frac{z_{0}}{L}\right)+G\left(\frac{Y}{L}, \frac{Z}{L}\right),
\end{aligned}
$$

equation (13) is merely the velocity defect law where $\frac{v_{\mathrm{L} \text { max }}}{u_{* 0}}$ has been expressed in terms of mean flow quantities.

We now use a standard procedure to evaluate the mean flow rate by integrating the velocity defect law right to the wall. In single phase flow, this procedure leads to very small errors in the determination of the friction coefficient since it neglects only the difference in velocity between the viscous and buffer layer law and the logarithmic law $[10,11]$. The higher the Reynolds number, the smaller the error. This technique is preferred to the integration of the inner law right to the maximum velocity location which leads to greater errors (Fig. 2); furthermore, all universal coefficients used so far have been determined by this procedure and it is likely that they will have comparable values in our case.

We thus obtain

$$
\begin{aligned}
\left\langle v_{\mathrm{L}}\right\rangle_{s_{\mathrm{L}}}=\frac{v_{\mathrm{L}}}{1-\alpha} & = \\
& =u_{* 0}\left[A \log \frac{u_{* 0} L}{v_{\mathrm{L}}}+B_{0}-C_{0}-D_{0}\right]
\end{aligned}
$$

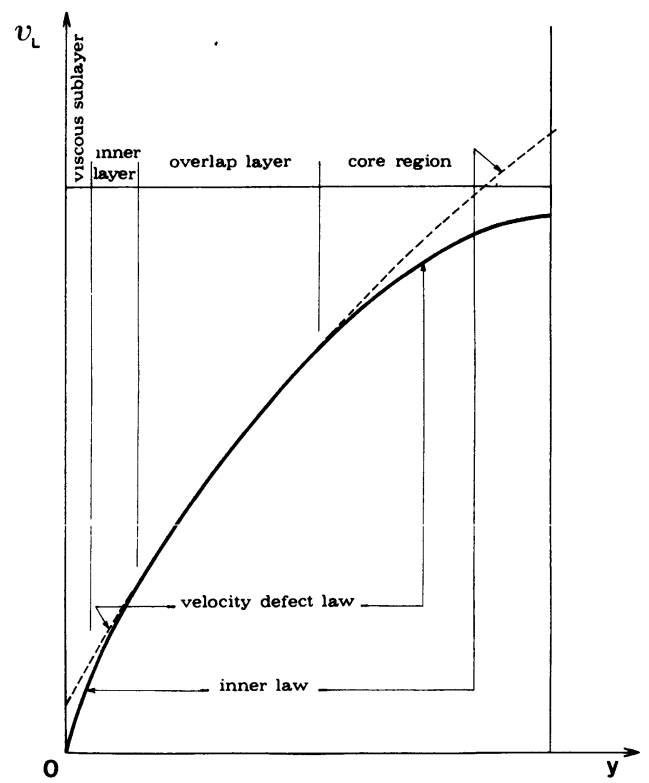

Fig. 2. - Integration of the velocity defect law. Comparison with exact profile. 
where

$$
C_{0}=C_{\mathrm{L}}\left(\frac{z_{0}}{L}\right)
$$

and

$$
D_{0}=-\frac{L^{2}}{S_{\mathrm{L}}} \iint_{S_{\mathrm{L}}} G_{\mathrm{L}}\left(\frac{Y}{L}, \frac{Z}{L}\right) \mathrm{d}\left(\frac{Y}{L}\right) \mathrm{d}\left(\frac{Z}{L}\right)
$$

are universal functions of $\alpha$ for a given cross-section. Further taking the length scale $L$ to be

$$
L=\frac{4 S_{\mathrm{L}}}{P_{\mathrm{L}}}
$$

and defining the friction coefficient of the liquid flow

$$
f_{\mathrm{L}}=\frac{2\left\langle\tau_{\mathrm{L}}\right\rangle_{P_{\mathrm{L}}}}{\rho_{\mathrm{L}}\left\langle v_{\mathrm{L}}\right\rangle_{S_{\mathrm{L}}}^{2}}=2\left(\frac{u_{* 0}(1-\alpha)}{u_{\mathrm{L}}}\right)^{2}
$$

and the liquid Reynolds number based upon mean flow properties

$$
R e_{\mathrm{L}}=\frac{4 Q_{\mathrm{L}}}{P \mu_{\mathrm{L}}}=\frac{U_{\mathrm{L}} D_{\mathrm{H}}}{v_{\mathrm{L}}}
$$

we get the final expression

$$
\sqrt{\frac{2}{f_{\mathrm{L}}}}=A \log R e_{\mathrm{L}} \sqrt{\frac{f_{\mathrm{L}}}{2}}+A \log \frac{P}{P_{\mathrm{L}}}+B_{0}-C_{0}-D_{0} .
$$

This expression is similar to Colebrook's formula but takes into account the variation of the wall stress along the wetted perimeter through the functions $C_{0}(\alpha)$ and $D_{0}(\alpha)$. The appearance of the ratio $P / P_{\mathrm{L}}$ is due to the use of the global rather than the effective Reynolds number and the choice of the length scales.

4. The gas phase region. - As previously indicated, we split the gas filled area of the cross-section into a region where the turbulent properties are controlled by the wall stress (subscript 1) and another where they are controlled by the interfacial stress (subscript 2). That the layer near the wavy interface can be scaled by the interfacial shear stress only is supported by the experiments of Suzanne [16], Tsacoyannis [17], Gayral [18].

Scaling the wall stress and the interfacial stress independently improves the accuracy of their evaluation, as can be expected from the work of Laufer [19]. Single scaling models, such as [6] and [9] are largely inaccurate, especially in upward flow; this is almost certainly due to the important dissymmetry of the shear stresses at the boundaries which can be taken into account only through proper scaling of the turbulent flow. It is not clear from the above cited results whether the introduction of a slip velocity at the boundary could increase the consistency of the model for this quantity appears to be small compared to the gas mean velocity in all cases examined so far, but it is used for the sake of completeness. There is a known problem for the choice of the origin of the transverse coordinate $y$ for the boundary layer over a rough interface. This problem is not solved at present and we shall assume that this origin is defined by the best fit of the universal logarithmic velocity profile above the wavy interface with experimental data.
The locus of these origins might not coincide with the mean interface position determined by the mass conservation laws, but to the present accuracy of the formulation it is probably irrelevant to distinguish the two surfaces.

We now use an identical method to that of the preceding section to evaluate the mean stress on the gas phase boundaries. The velocity defect law is shown to be logarithmic, i.e.

$$
\frac{v_{\mathrm{G}}-v_{\mathrm{G} \max }}{u_{* \mathrm{G}_{0}}}=G_{1}\left(\frac{Y}{L_{1}}, \frac{Z}{L_{1}}\right)
$$

should be written as

$$
\frac{v_{\mathrm{G}}-v_{\mathrm{G} \max }}{u_{* \mathrm{G}}(z)}=A \log \frac{y}{L_{1}}+C_{\mathrm{G}_{1}}\left(\frac{z}{L_{1}}\right)
$$

in the wall coordinates as it is matched with the inner law

$$
\frac{v_{\mathrm{G}}}{u_{* \mathrm{G}}(z)}=A \log \frac{y u_{* \mathrm{G}}(z)}{v_{\mathrm{G}}}+B_{1}
$$

in the overlap region.

We define

$$
\begin{aligned}
L_{1} & =\frac{4 S_{1}}{P_{\mathrm{G}}} \\
u_{* \mathrm{G}_{0}}^{2} & =\frac{\left\langle\tau_{\mathrm{G}}\right\rangle_{P_{\mathrm{G}}}}{\rho_{\mathrm{G}}}=\left\langle u_{* \mathrm{G}}^{2}\right\rangle_{P_{\mathrm{G}}} \\
f_{\mathrm{G}} & =\frac{2\left\langle\tau_{\mathrm{G}}\right\rangle_{P_{\mathrm{G}}}}{\rho_{\mathrm{G}}\left\langle v_{\mathrm{G}}\right\rangle_{S_{1}}^{2}} \\
R e_{\mathrm{G}} & =\frac{4 Q_{\mathrm{G}}}{P \mu_{\mathrm{G}}}=\frac{v_{\mathrm{G}} D_{\mathrm{H}}}{v_{\mathrm{G}}}
\end{aligned}
$$


and write (19) and (20) at the point $z_{1}$ where

$$
u_{* \mathrm{G}}\left(z_{1}\right)=u_{* \mathrm{G}_{0}} v_{\mathrm{G} \max }
$$

is eliminated by substraction. Integrating the velocity defect law right to the wall, as previously explained, gives :

$$
\begin{aligned}
\sqrt{\frac{2}{f_{\mathrm{G}}}} & =A \log R e_{\mathrm{G}} \sqrt{\frac{f_{\mathrm{G}}}{2}}+A \log \frac{P}{P_{\mathrm{G}}}+ \\
& +A \log \frac{\left\langle v_{\mathrm{G}}\right\rangle_{S_{1}}}{\left\langle v_{\mathrm{G}}\right\rangle_{S_{\mathrm{G}}}} \cdot \frac{S_{1}}{S_{\mathrm{G}}}+B_{1}-C_{1}-D_{1}
\end{aligned}
$$

where

$$
C_{1}=C_{\mathrm{G}_{1}}\left(\frac{z_{1}}{L_{1}}\right)
$$

and

$$
D_{1}=-\frac{L_{1}^{2}}{S_{1}} \iint_{S_{1}} G_{1}\left(\frac{Y}{L_{1}}, \frac{Z}{L_{1}}\right) \mathrm{d}\left(\frac{Y}{L_{1}}\right) \mathrm{d}\left(\frac{Z}{L_{1}}\right)
$$

are universal functions of $\alpha$ for a given cross-section shape ; $B_{1}$ is a function of the wall roughness.

For the remainder of the gas-filled cross-section $S_{2}$, we follow similar lines apart from the introduction of a slip velocity $v_{\varepsilon}$ at the mean interface to account for the motion of the waves. We also define

$$
\begin{aligned}
L_{2} & =\frac{4 S_{2}}{P_{i}} \\
u_{* i_{0}}^{2} & =\frac{\left\langle\tau_{i}\right\rangle_{P_{i}}}{\rho_{\mathrm{G}}}=\left\langle u_{* i}^{2}\right\rangle_{P_{i}}
\end{aligned}
$$

and

$$
f_{i}=\frac{2\left\langle\tau_{i}\right\rangle_{P_{i}}}{\rho_{\mathrm{G}}\left(\left\langle v_{\mathrm{G}}\right\rangle_{S_{2}}-v_{\varepsilon_{0}}\right)^{2}}
$$

and an abscissae $z_{2}$ where

$$
u_{* i}\left(z_{2}\right)=u_{* i_{0}}
$$

and

$$
v_{\varepsilon}\left(z_{2}\right)=v_{\varepsilon_{0}} \text {. }
$$

Writting the velocity defect law

$$
\frac{v_{\mathrm{G}}-v_{\mathrm{G} \max }}{u_{* i_{0}}}=G_{2}\left(\frac{Y}{L_{2}}, \frac{Z}{L_{2}}\right)
$$

as a logarithmic profile in boundary coordinates

$$
\frac{v_{\mathrm{G}}-v_{\mathrm{G} \max }}{u_{* i}(z)}=A \log \frac{y}{L_{2}}+C_{\mathrm{G}_{2}}\left(\frac{z}{L_{2}}\right)
$$

in the region where it overlaps the inner law

$$
\frac{v_{\mathrm{G}}-v_{\varepsilon}(z)}{u_{* i}(z)}=A \log \frac{y u_{* i}(z)}{v_{\mathrm{G}}}+B_{\mathrm{G}_{2}}
$$

gives, as before, the velocity defect law in terms of mean flow quantities and

$$
\begin{aligned}
\sqrt{\frac{2}{f_{i}}}= & A \log R e_{\mathrm{G}} \sqrt{\frac{f_{i}}{2}}+A \log \frac{P}{P_{i}}+ \\
& +A \log \frac{\left\langle v_{\mathrm{G}}\right\rangle_{S_{2}}}{\left\langle v_{\mathrm{G}}\right\rangle_{S_{\mathrm{G}}}} \cdot \frac{S_{2}}{S_{\mathrm{G}}}+B_{2}-C_{2}-D_{2}
\end{aligned}
$$

$B_{\mathrm{G}_{2}}$ depends on the equivalent uniform sand roughness $\varepsilon$ of the interface at abscissae $z$ and

$$
B_{2}=B_{\mathrm{G}_{2}}\left(\varepsilon\left(z_{2}\right)\right), \quad \text { or } \quad B_{2}=B_{\mathrm{G}_{2}}\left(\varepsilon_{0}\right)
$$

where

Also

$$
\varepsilon_{0}=\varepsilon\left(z_{2}\right)
$$

$$
C_{2}=C_{\mathrm{G}_{2}}\left(\frac{z_{2}}{L_{2}}\right)
$$

and

$$
D_{2}=-\frac{L_{2}^{2}}{S_{2}} \iint_{S_{2}} G_{2}\left(\frac{Y}{L_{2}}, \frac{Z}{L_{2}}\right) \mathrm{d}\left(\frac{Y}{L_{2}}\right) \mathrm{d}\left(\frac{Z}{L_{2}}\right) .
$$

$C_{2}$ and $D_{2}$ should be universal functions of $\alpha$ for a given cross-section profile, $B_{2}$ is a function of the properties of the fluids and flow parameters since it reflects the transfer of momentum from the gas to the liquid via the wave (or ripple) process.

5. The closure relationships. - 5.1 THE CHOICE OF $S_{1} / S_{2}$. - Only a correct choice of the boundary between $S_{1}$ and $S_{2}$ can assure the universality of the functions $C_{1}(\alpha), D_{1}(\alpha), C_{2}(\alpha), D_{2}(\alpha)$. Ideally, a surface of minimum stress could be chosen, but in most cases this would entrain the solving of the whole turbulent flow problem. However, we feel that a reasonable choice of the ratio $S_{1} / S_{2}$ can be made on semitheoretical grounds.

Experiments in rectangular channels of high aspect ratio (Laufer [19]) or in other type of flow where plane symmetry is likely to be prevalent (Suzanne [16]) show that the shear stress varies linearly with the crossstream coordinate and is very nearly zero at the point of maximum velocity. In this case, the obvious choice for the area ratio is, neglecting the small distance between zero stress and velocity maximum locations

$$
\frac{S_{1}}{S_{2}}=\left(\frac{u_{* \mathrm{G}_{0}}}{u_{* i_{0}}}\right)^{2}
$$

On the other hand, in the case of a motionless interface $f_{i} \simeq f_{\mathrm{G}}$ holds provided that $\left\langle\tau_{\mathrm{G}}\right\rangle_{P_{\mathrm{G}}} \simeq\left\langle\tau_{i}\right\rangle_{P_{i}}$ and $D_{1} \simeq D_{2}$, as expected in the limit of very high Reynolds number (Fortier [10], Coles [20]). The identity of (25) and (32) then implies $S_{1} / S_{2}=P_{\mathrm{G}} / P_{i}$. 
The above remarks suggest the following interpolation formula for the area ratio in the general case

$$
\frac{S_{1}}{S_{2}}=\frac{P_{\mathrm{G}}}{P_{i}}\left(\frac{u_{* \mathrm{G}_{0}}}{u_{* i_{0}}}\right)^{2}
$$

which should give the correct answer in the two limit cases cited above.

It should be reminded that the main purpose of the above semi-empirical relation (33) is to evaluate the flow integral over the cross sectional area of the gas and that a slight error in the prediction of the locus of the maximum gas velocity is acceptable. Thus it is somewhat illusory to use a more precise turbulence model at present though this particular closure relationship can be improved in the future.

5.2 THE UNIQUENESS OF $v_{\mathrm{G} \max }$. - Both core regions in $S_{1}$ and $S_{2}$ have to be matched. This is achieved by using the same value of $v_{\mathrm{G} \text { max }}$ in both regions (and correct values of $D_{1}$ and $D_{2}$ ).

5.3 The MEAN INTERFACE PROFILE. - In principle, the mean interface profile should be an outcome of the theory. It can be found by solving a transverse momentum balance equation taking into account the secondary flow in the gas phase. Some attempts have been made in this field especially for the prediction of horizontal annular flow (Butterworth [21]), but the full analysis of the mechanisms involved has yet to be achieved. In view of the comparison of our theory with laboratory experiments, the mean interface profile can be given a priori; the values of $P_{\mathrm{G}}, P_{\mathrm{L}}, P_{i}$ are then prescribed algebraic functions of $\alpha$. This procedure is correct for small upward angles $\beta \lesssim 10^{\circ}$ and low density ratio as in air-water flow at standard pressure.

5.4 The UNIVERSAL FUNCTIONS. - All the quantities $A, B_{0}, C_{0}, D_{0}, B_{1}, C_{1}, D_{1}, C_{2}, D_{2}$ should be prescribed. $A$ is a universal constant. $B_{0}$ and $B_{1}$ are function of Reynolds numbers based on roughness height and friction velocities and can be estimated from single phase flow pressure drop measurements in the same channel. $C_{0}, D_{0}, C_{1}, D_{1}, C_{2}, D_{2}$ are universal functions of $\alpha$ for a given cross-section and could be obtained by anemometry and local shear stress measurements. As no such data is available at present, we shall deduce their value from single phase flow analogy. Suzanne [16]'s results have been found insufficients to evaluate the mentionned constants.

5.5 THE INTERFACE CHARACTERISTIC FUNCTIONS. $B_{2}$ is a function of a Reynolds number based on the roughness height of the interface and mean friction velocity

$$
B_{2}=-A \log \frac{\varepsilon_{0} u_{* i_{0}}}{v_{\mathrm{G}}}+B_{2}^{\prime} .
$$

Assuming all other (universal) constants are known, $\varepsilon_{0}$ is the only physical characteristic of the model. In principle, it depends upon geometrical and flow properties, including $\alpha$, and the physical properties of both fluids and the interface. However, though several authors (Charnock [22], Hidy and Plate [23], Wu [24], Phillips [25], Theofanous [26] Lilleleht [27], Larson and Wright [28], Suzanne [16], Moeck and Stachiewicz [29]) have proposed expressions for $\varepsilon_{0}$, they are far from falling into agreement. At this stage of analysis, it is then better to take $\varepsilon_{0}$ as a free parameter to be evaluated from experiments, with the exception of one set of data giving both the values of $\varepsilon_{0}$ and pressure gradient so that direct comparison can be made [16]. It will be shown in the next section that direct comparison of the model with experimental data gives reasonable values of $\varepsilon_{0} . v_{\varepsilon_{0}}$ is the absolute velocity of the waves ensuring most of the momentum transfer between phases. In the laboratory experiments so far examined, these waves have a short wave-length and propagate at low velocity compared to the gas mean flow velocity. A constant small velocity ratio $\frac{v_{\varepsilon_{0}}}{v_{\mathrm{G} \text { max }}}$ has been used in the test procedure as in Moeck and Stachiewicz [29].

6. Comparison with experiments and discussion. The above described model has been tested by comparison with two sets of data. One is given by Suzanne [16] and was obtained in a rectangular channel of crosssection $0.1 \times 0.2 \mathrm{~m}^{2}$ with a downward slope of $0.08 \%$. The other is given by Crouzier [9] for a circular duct of I.D. $0.045 \mathrm{~m}$ and upward slopes of $0 \%, 2 \%$ and $5 \%$. Both authors used air and water at atmospheric pressure. The first author measured velocity profiles in both phases and deduced the equivalent sandroughness of the interface from gas anemometry. Both authors give pressure drop and liquid fraction in the well developed flow sections of the channels.

In both experiments, the mean interface was almost plane and this assumption was introduced in our calculations. There is still some controversy on the values of the universal logarithmic profiles constants $A$ and $B$. Though the constant $A$ seems to increase with Reynolds number (Tennekes and Lumley [12]), we have taken $A=2.46$ as pertinent to the Reynolds number range used in [6] and [9]. Also, most of the evaluations of the second constant are made using this value and it should be kept for the sake of coherency. Owing to the lack of measured values of the $C_{i}$ and $D_{i}, i=0,1,2$ functions in stratified flow, we have been guided by single phase values in our choice for the comparison test.

Fortier [10] gives $D=4.44$ for a circular duct, a value very close to that of Laufer [30] :4.46. With our chosen value of the constant $A$, this implies $C=0.96$. Comparison with the Von Karman-Nikuradze formula gives $B=5.69$ in good agreement with experimental data.

In the limit case of small liquid holdup, two dimen- 
Table I.

Function

Circular duct

Two-dimensional flows

Values of the universal functions deduced from single phase flow analogy

sional analysis of the liquid layer is possible and gives (assuming a constant value of the shear stress)

$$
C_{0}+D_{0}=A\left(\log \frac{2}{3}+\frac{8}{3}\right)+\mathcal{O}\left(\alpha_{\mathrm{L}}^{2}\right)=5.56 \text {. }
$$

The value is 5.4 for $\alpha=0$.

We have thus used the simple following interpolation formula for arbitrary $\alpha$ :

$$
C_{0}+D_{0}=5.40+0.16\left(2 \alpha-\alpha^{2}\right) .
$$

In two-dimensional flow, there is some discrepancy between the values of $D$ given by Fortier [10] : 2.34 and Laufer [19] : 3.15. Following the same lines as above, we have chosen the values shown in table $I$. $\varepsilon_{0} / D_{\mathrm{H}}$ has been used as a free parameter for comparison purposes except for the data of Suzanne [16] where measured values of $\varepsilon_{0}$ were available.

$v_{\varepsilon_{0}} / v_{\mathrm{G} \max }$ was kept constant and equal to 0.07 .

Figures 3 and 4 show that most of the data of Suzanne is well predicted by taking a value of $\varepsilon_{0} / D_{H}$ of 0.05 . This implies (Cohen and Hanratty [31]) a wave height of the order of $0.0015 \mathrm{~m}$ in agreement with the observations.

Using now the values of $\varepsilon_{0} / D_{\mathrm{H}}$ deduced from the velocity profile measurements, very good agreement is obtained between theory and experiment (Figs. 5

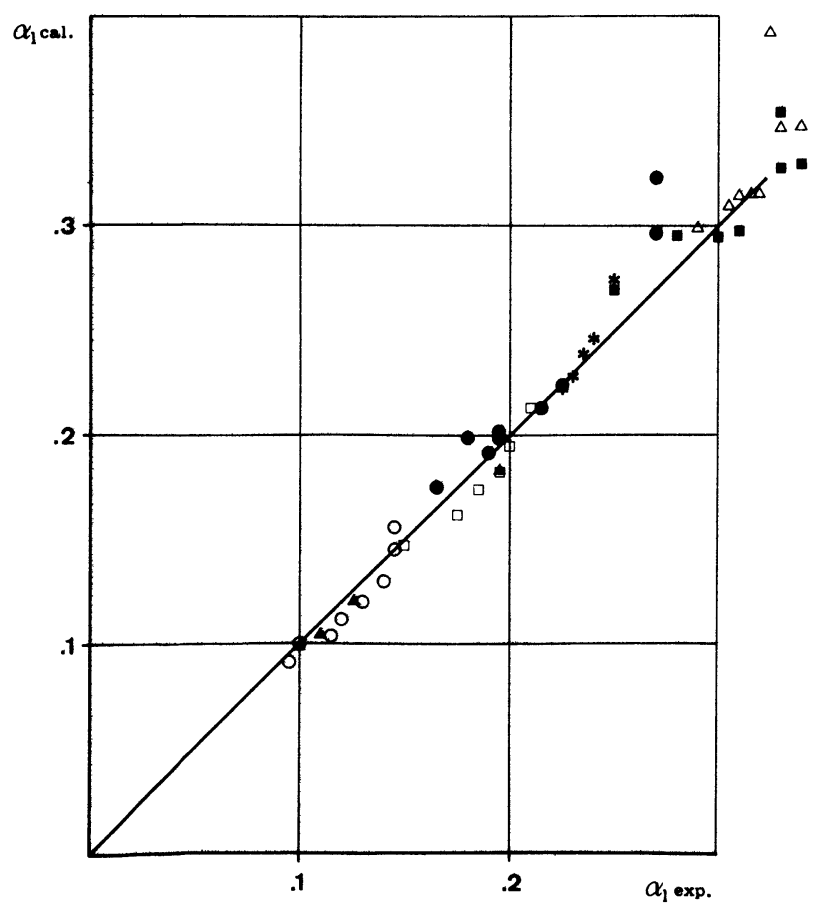

Fig. 3. - Experimental liquid fraction from Suzanne [16] compared with theory $\left(\varepsilon_{0} / D_{\mathrm{H}}=0.05\right)$.

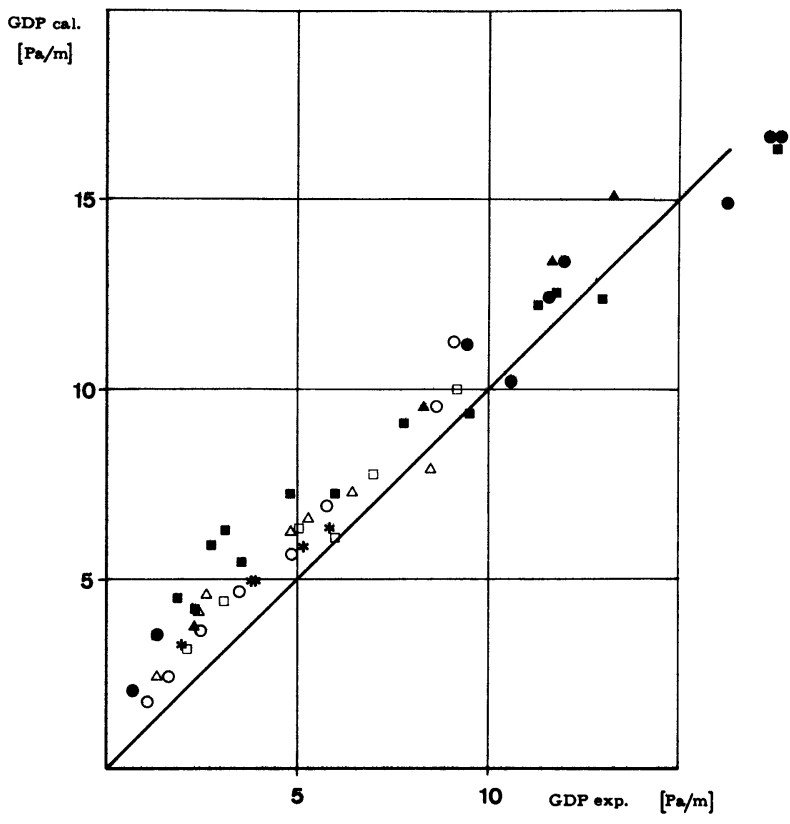

Fig. 4. - Experimental pressure gradient from Suzanne [16] compared with theory $\left(\varepsilon_{0} / D_{\mathrm{H}}=0.05\right)$.

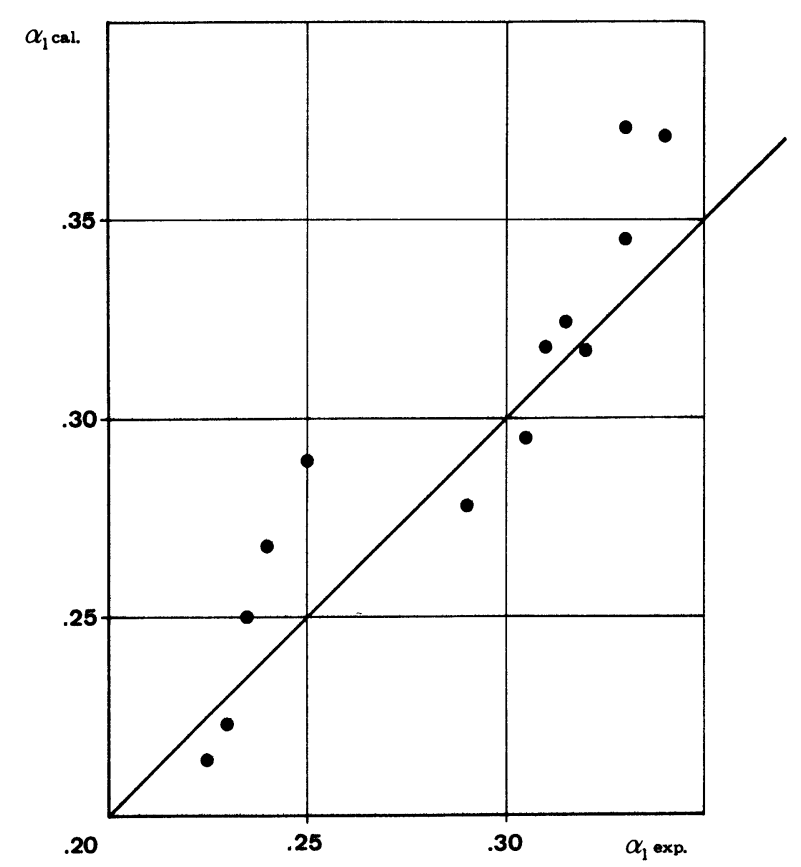

Fig. 5. - Experimental liquid fraction from Suzanne [16] compared with theory $\left(\varepsilon_{0} / D_{\mathrm{H}}\right.$ evaluated from velocity profiles). 
and 6). No values of $\varepsilon_{0} / D_{\mathrm{H}}$ were available in the case of Crouzier [9]. Figures 7 to 10 show the measured liquid fraction and pressure gradient for a set of gas and liquid flow rates for three slopes $(0 \%, 2 \%$ and $5 \%$, upward) and the theoretical calculations for $\varepsilon_{0} / D_{\mathrm{H}}=0,0.05$ and 0.20 . It is seen that a single value of $\varepsilon_{0} / D_{\mathrm{H}}$ allows good prediction of both quantities.

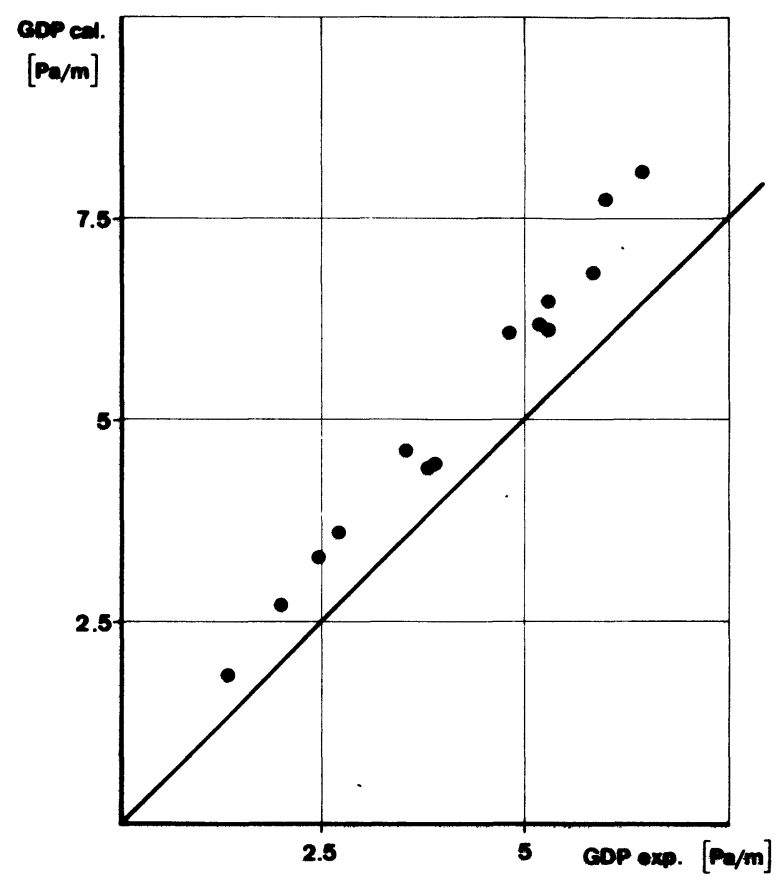

Fig. 6. - Experimental pressure gradient from Suzanne [16] compared with theory $\left(\varepsilon_{0} / D_{\mathrm{H}}\right.$ evaluated from velocity profiles).

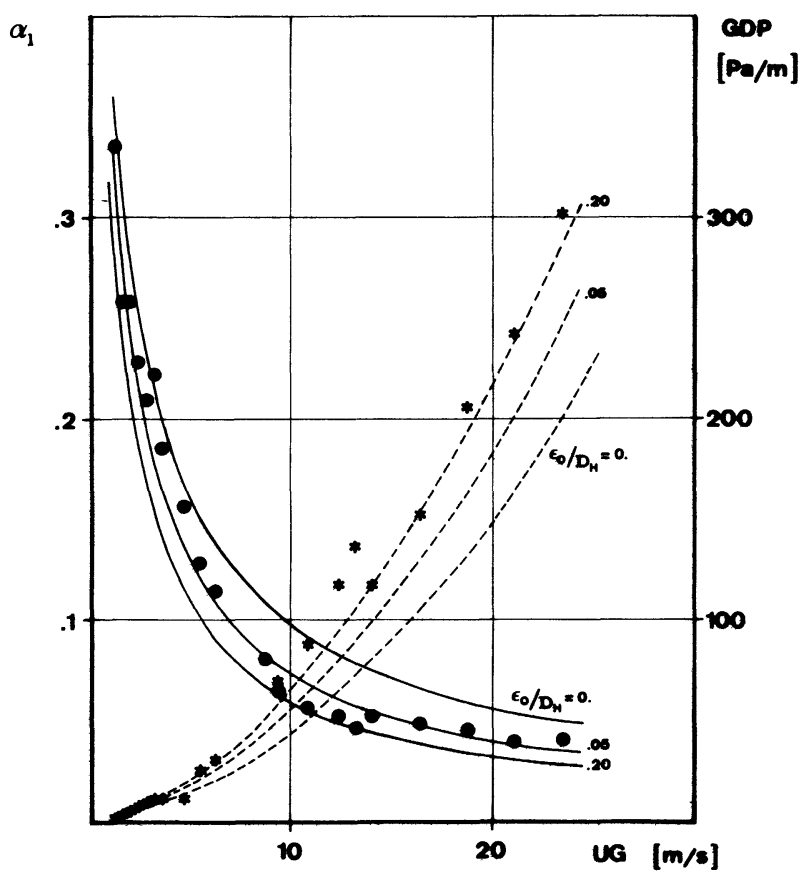

Fig. 7. - Comparison of experimental (Crouzier [6]), and theoretical liquid fraction and pressure gradient. $U_{\mathrm{L}}=0.0311 \mathrm{~m} / \mathrm{s}$; $\beta=0.0$.
This is felt to be a considerable improvement upon previous models. Also, it does not vary with slope for identical internal conditions, as expected from basic considerations.

The values of $\varepsilon_{0} / D_{\mathrm{H}}$ that fit the data are relatively independent of the gas rate for a given liquid rate. This is not paradoxical since the liquid fraction

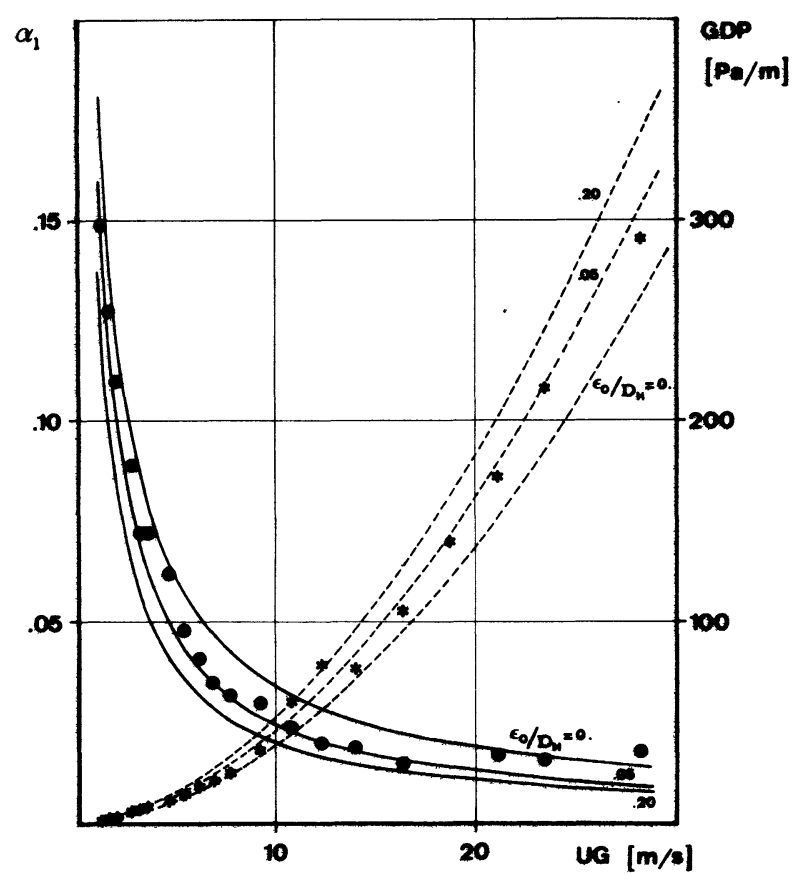

Fig. 8. - Comparison of experimental (Crouzier [6]) and theoretical liquid fraction and pressure gradient. $U_{\mathrm{L}}=0.0083 \mathrm{~m} / \mathrm{s}$; $\beta=0.0$.

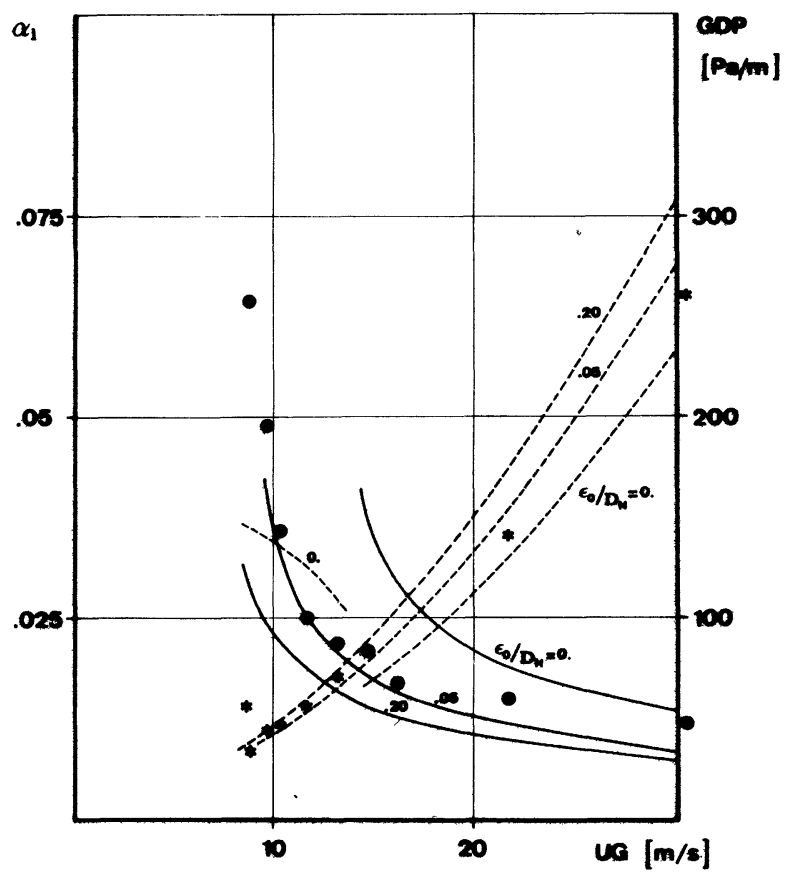

Fig. 9. - Same as figure 7 with $\operatorname{tg} \beta=0.02$. 


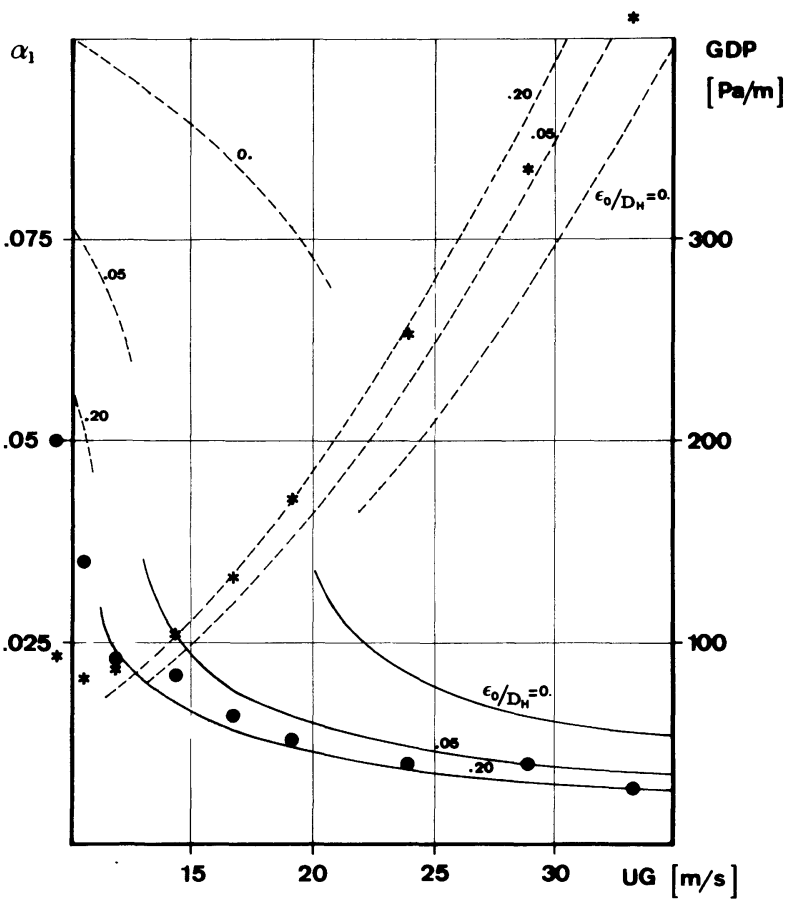

Fig. 10. - Same as figure 7 with $\operatorname{tg} \beta=0.05$.

diminishes as the gas rate is increased so that one effect compensates the other. The results are thus coherent with the usually observed increase of the gas-liquid friction with gas velocity and liquid fraction (Moeck and Stachiewicz [29], Crouzier [9], Wallis [32]).

To predict pressure drop and liquid content in any pertinent situation, one requires an interfacial roughness estimate. The present number and extent of experiments is too small to give any precise law valid over most of the current physical parameters range. However, in situations where the momentum transfer is similar to that of the already cited experiments, a modified Charnock law [22]

$$
\varepsilon_{0}=k \alpha_{\mathrm{L}} \frac{u_{* i}^{2}}{g}
$$

seems to give satisfying results. This is shown in figures $11 a$ and $11 b$ for near horizontal flow with the value $k=1$ taken as a tentative closure assumption.

The validity of (35) is probably fortuitous and it should be used with caution until more experimental evidence can be gained.

7. Conclusion. - The model presented for the calculation of pressure drop and liquid fraction in fully developed stratified turbulent gas-liquid flow is based on the properties of high Reynolds number wall bounded turbulent flow. Its mathematical expression depends on the geometry of the cross-section only through six functions of the liquid fraction. Although they are yet to be measured, approximate values have been estimated by analogy with single phase flows for the sake of comparison of the theory with expe-

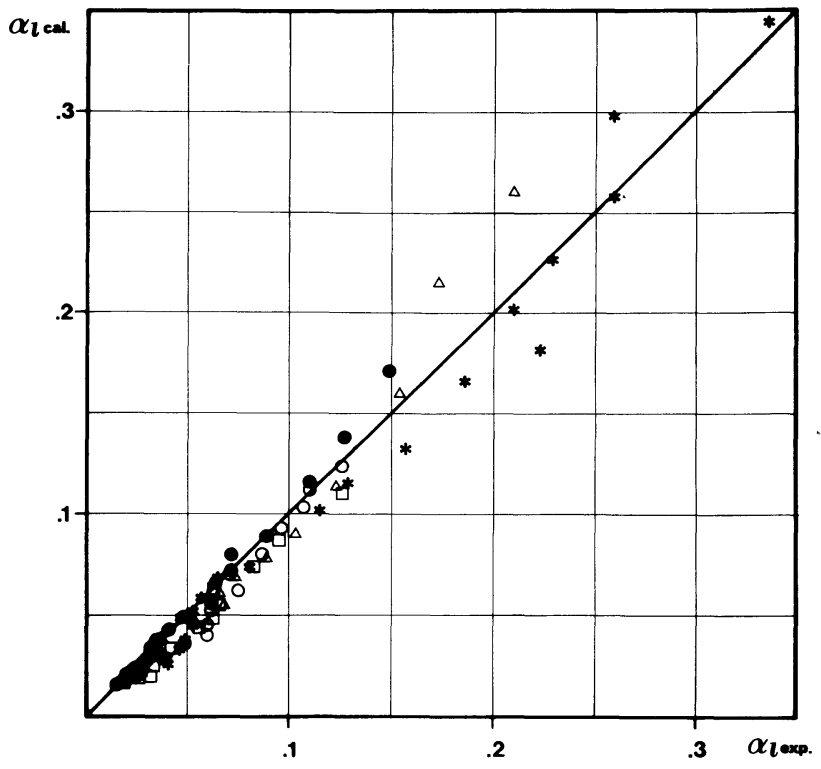

a)

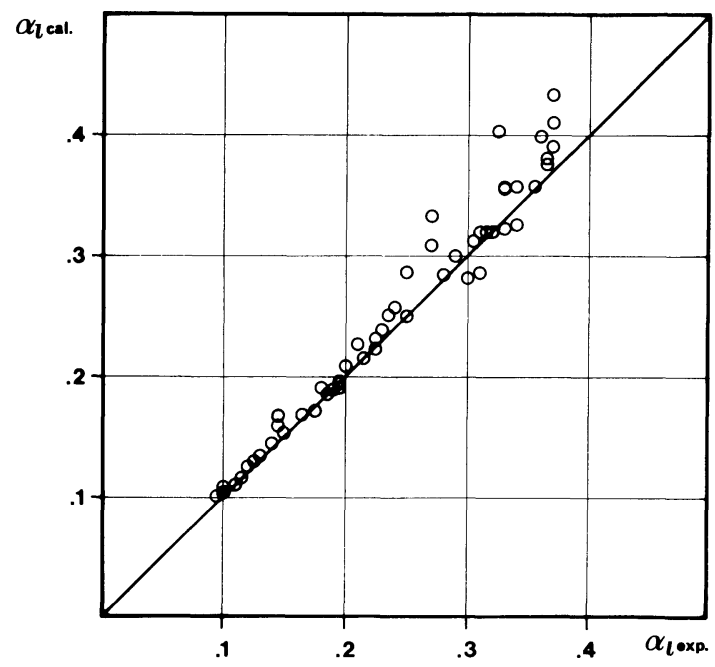

b)

Fig. 11. - Comparison of experimental ( $(a)$ : Crouzier [9] and (b) Suzanne [16]) with theoretical liquid fraction using

$$
\varepsilon_{0}=\alpha_{\mathrm{L}} \frac{u_{* i}^{2}}{g}
$$

rimental data. The remaining input is the roughness height of the wavy interface with respect to the gas flow. It can be adjusted to give good prediction of both the liquid fraction and the pressure gradient for the test cases considered in this article and its value is found to be in close agreement with other authors evaluation independently of the slope or the geometry of the cross-section.

Although the first results are encouraging, the authors feel that comparison with other data obtained in widely different experimental conditions, especially with various effluents and absolute pressure values is needed though they are scarce at present. 
Furthermore, accurate anemometry in both phases in laboratory set ups could be used to measure the remaining unknown functions, thus enabling the precise evaluation of the wavy interface roughness parameters. Such a work is highly recommended.

Simpler models can be used, for instance with single scaling in the gas phase and fewer friction laws constants. However, the gain could be only apparent since these constants would have lost universality and depend on all flow and physical parameters for which correlations should be provided by time consuming experimental work.

Acknowledgments. - This work has been undertaken under a grant CNRS-ATP 4143. Anterior material on which part of this work is based has been sponsored by SNEA(P), CFP and IFP.

Appendix. - The time averaged form of the phase mass and $X$-wise momentum conservation theorems are used throughout the text using the simplified notation described below. It is also understood that the mass averaged velocity is used instead of the time averaged one, so that density fluctuations are automatically included in the definitions $[13,33]$.

The mass conservation theorem [14] reads

$$
Q_{\mathrm{G}}=S\left\langle\alpha_{\mathrm{G}} \overline{\overline{\rho_{\mathrm{G}} v_{\mathrm{G}}}}\right\rangle_{S}=S\left\langle\alpha_{\mathrm{G}} \overline{\bar{\rho}}_{\mathrm{G}}{\tilde{v_{\mathrm{G}}}}_{\rangle_{S}}\right.
$$

or

$$
Q_{\mathrm{G}}=S\left\langle\alpha_{\mathrm{G}} \rho_{\mathrm{G}} v_{\mathrm{G}}\right\rangle_{S}
$$

in simplified notation.
It can be assumed that the mean gas density vary very little over the entire cross-section and, thus we write

$$
\frac{Q_{\mathrm{G}}}{\rho_{\mathrm{G}} S}=\left\langle\alpha_{\mathrm{G}} v_{\mathrm{G}}\right\rangle_{s}=U_{\mathrm{G}} .
$$

We now chose a surface $S_{\mathrm{G}}$ (defining the mean interface) so that

$\int_{S} \alpha_{\mathrm{G}} v_{\mathrm{G}} \mathrm{d} S=\int_{S_{\mathrm{G}}} v_{\mathrm{G}} \mathrm{d} S$ and put $\alpha=\frac{S_{\mathrm{G}}}{S}$

Then (A.2) can be written

$$
U_{\mathrm{G}}=\alpha\left\langle v_{\mathrm{G}}\right\rangle_{S_{\mathrm{G}}} \quad \text { (exact equation). }
$$

From mean value theorems, we have

$$
\frac{\left\langle\alpha_{\mathrm{G}}\right\rangle}{\alpha}=\frac{\left\langle v_{\mathrm{G}}\right\rangle_{S_{\mathrm{G}}}}{\left\langle v_{\mathrm{G}}\right\rangle_{1}}
$$

where

$$
\left\langle v_{\mathrm{G}}\right\rangle_{1}=\frac{\int_{S} \alpha_{\mathrm{G}} v_{\mathrm{G}} \mathrm{d} S}{\int_{S} \alpha_{\mathrm{G}} \mathrm{d} S}
$$

is a weighted mean value of the velocity.

The ratio (A.5) tends to unity when the wavy zone reduces to zero thickness or when $v_{\mathrm{G}}$ tends to zero in the wavy zone. We can expect that in most application the definition (A.3) of $S_{\mathrm{G}}$ leads to small errors in the determination of $\left\langle\alpha_{G}\right\rangle$. Thus we have confounded in the text $\alpha$ and $\left\langle\alpha_{G}\right\rangle$ and the mean values of wetted perimeters with their definition from $S_{\mathrm{G}}$.

\section{References}

[1] Govier, G. W. and AzIz, K., Flow of Complex Mixtures in Pipes (Van Nostrand, Reinhold Co., New York) 1972.

[2] Bonnin, J. and FitremanN, J. M., Special Number Houille Blanche (1978).

[3] Yu, H. S. and Sparrow, E. M., AIChE J. 13 (1967) 10.

[4] Flanigan, O., Oil Gas J. March 10 (1958) 132.

[5] Eaton, B. A., Andrews, D. E., Knowles, C. R., SilberberG, I. H. and Brown, K. E., J. Pet. Technol. (1967) 815.

[6] TAitel, Y. and Dukler, A. E., AIChE J. 22 (1976) 47.

[7] Russell, T. W. F., Etchells, A. W., Jensen, R. H. and Arruda, P. J., AIChE J. 20 (1974) 664.

[8] Cheremisinoff, N. P. and Davis, E. J., AIChE J. 25 (1979) 48.

[9] Crouzier, O., Thesis, Univ. of Paris VI (1978).

[10] ForTIER, A., Mécanique des Fluides et Transferts de chaleur et de masse par convection (Masson, Paris) 1976.

[11] Hinze, J. O., Turbulence (Mc Graw Hill Co., New York) 1975.

[12] Tennekes, H. and Lumley, J. L., A First Course in Turbulence (MIT Press) 1972.

[13] IsHII, M., Thermo-Fluid Dynamic Theory of the Two-phase Flow (Ed. Eyrolles) 1975.

[14] Fitremann, J. M., Thesis, Univ. of Paris VI (1977).

[15] Delhaye, J. M. and AChaRD, J. L., CSNI, Specialists Meeting on Transient Two-phase Flow, Toronto (1976).

[16] Suzanne, C., Thesis, Univ. of Toulouse (1977).

[17] Tsacoyannis, J., Thesis, Univ. of Toulouse (1976).
[18] Gayral, B., Thesis, Univ. of Toulouse (1979).

[19] Laufer, J., Nat. Advis. Comm. Aero., Wash., Rep. no 1053 (1951).

[20] Coles, D., J. Fluid Mech. 1 (1956) 191.

[21] Butterworth, D., Progress in Heat and Mass Transfer, Proceedings of the International Symposium on TwoPhase systems 6 (Pergamon, Haifa) 1971, 235.

[22] Charnock, H., Q. J. R. Meteorol. Soc. 81 (1955) 639.

[23] Hidy, G. M. and Plate, E. J., J. Fluid Mech. 10 (1961) 189.

[24] Wu, J., J. Fluid Mech. 61 (1973) 275.

[25] PhilliPs, O. M., The dynamics of the upper ocean (Cambridge University Press, London) 1966.

[26] Johns, D. M., Theofanous, T. G. and Houze, R. N., Proceedings of the third Symposium on Turbulence in Liquids, Eng. Univ. of Missouri-Rolla (1973) 250.

[27] Lilleleht, L. U. and HaNRATTy, T. J., AIChE J. 7 (1961) 548.

[28] LARson, T. R. and Wright, J. W., J. Fluid Mech. 70 (1975) 417.

[29] Moeck, E. O. and Stachiewicz, J. W., J. Heat Mass Transfer 15 (1972) 637

[30] Laufer, J., Nat. Advis. Comm. Aero., Wash., Rep. no 1174 (1954).

[31] Cohen, L. S. and Hanratty, T. J., J. Fluid Mech. 31 (1968) 467.

[32] Wallis, G. B., One-dimensional Two-Phase Flow (Mc Graw Hill, New York) 1969.

[33] FaVre, A., J. Méc. 4 (1965) 361. 